\title{
Un generador aleatorio de microestructuras virtuales 3D
}

\author{
A. $\operatorname{Martín}^{(*)}$, M. Tellaeche ${ }^{(*)}$ y J. Gil-Sevillano ${ }^{(*)}$ \\ Resumen Se describe un programa de ordenador que genera microestructuras virtuales en 3D. El sólido (nor- \\ malmente un cubo) se descompone en vóxeles, en los que se distribuye al azar un número prescrito \\ de semillas de "granos". El crecimiento de estas semillas puede activarse desde el principio de la \\ simulación o, gradualmente, durante el proceso de crecimiento de los granos. El programa dispone de \\ dos formas de asignar los vóxeles a un grano en concreto que se describen detalladamente. El progra- \\ ma reconoce los vóxeles situados en los límites de grano. Esto permite realizar fácilmente algunos \\ cálculos estereológicos o resembrar semillas en los bordes de grano y simular posteriores recristaliza- \\ ciones o transformaciones de fase nucleadas en intercaras.
}

Palabras clave: Microestructura. Estereología. Teselaciones. Transformaciones en fase sólida. Recristalización.

\section{Random generator of virtual 3D microstructures}

\begin{abstract}
A computer model for the generation of 3D grain microstructures is described. The solid (usually a cube) is discretised in voxels. Grain seeds are distributed at random in some voxels. These seeds can be placed beforehand or simultaneously with the grain growth process. There are two ways for assigning a voxel to a particular grain, which are described in detail. After completion of a 3D tessellation, the model identifies the voxels at the grain boundaries. This feature allows for an easy computation of stereological parameters or as a basis for simulating recrystallisation or phase transformations nucleated at grain boundaries.
\end{abstract}

Keywords: Microstructure. Stereology. Tessellations. Solid phase transformations. Recrystallisation.

\section{INTRODUCCIÓN}

Las microestructuras granulares generadas por ordenador son muy efectivas para comprender procesos metalúrgicos como solidificación o transformaciones en fase sólida. No existe ambigüedad sobre lo que es un grano o no lo es. En muchas ocasiones, puede interesar conocer el efecto que produce la introducción de un número mayor de semillas para la nucleación de los granos o introducir partículas que controlen su crecimiento, los efectos de una modificación en las velocidades de enfriamiento o la nucleación heterogénea de granos recristalizados o nuevas fases a partir de bordes de grano o de puntos triples. La simulación mediante ordena-

(*) Centro de Estudios e Investigaciones Técnicas de Guipúzcoa (CEIT) $\mathrm{P}^{\circ}$ de Manuel Lardizábal, 15, 20009 - San Sebastián y E.S. de Ingenieros (Univ. de Navarra), Aptdo. 1674, 20080 - San Sebastián (España). dor permite obtener una respuesta rápida y fiable a estas preguntas, a partir de leyes de proceso radicalmente simples, o puede utilizarse como herramienta para averiguar las distribuciones de tamaño de grano en diferentes condiciones de nucleación y crecimiento, proporción de bordes de grano relativa al volumen, densidad de puntos triples, etc. (1-4). Las microestructuras virtuales pueden servir también como base para el estudio numérico de otros procesos físicos (mecánicos, eléctricos, magnéticos, etc.), particularmente mediante el MEF.

\section{DESCRIPCIÓN DEL PROGRAMA}

El sólido (habitualmente un cubo) se descompone en un número prefijado de elementos cúbicos de volumen (vóxeles, v.g., $160 \times 160 \times 160$ ). Se atribuye a un cierto número de ellos el carácter de semillas o núcleos de grano. No se permite el 
solapamiento de semillas, esto es, una semilla nunca se readjudica a un vóxel ya designado previamente como tal.

El programa permite realizar dos tipos de teselaciones distintas del volumen, que a continuación se describen.

\subsection{Teselación determinista}

La teselación es unívoca si, una vez distribuidas las semillas y definida la norma para la medida de las distancias desde los vóxeles a las semillas, para cada vóxel del volumen se calculan las distancias a todas las semillas y el vóxel se asigna al grano cuya semilla esté a la mínima distancia. Las distancias pueden ser cartesianas (norma euclídea), en cuyo caso se obtiene una teselación de Voronoi en 3D (Fig. 1), con fronteras planas entre los granos.

Si se admite simultaneidad de nucleación y crecimiento de los granos, a la distancia verdadera se le resta el crecimiento que ha experimentado la semilla en cuestión, de acuerdo con la ley de crecimiento prescrita y el tiempo transcurrido desde que se generó esa semilla. El resultado es, entonces, una teselación de Johnson-Mehl-Avrami-Kolmogorov (JMAK)(5) y, ahora, los límites entre granos son paraboloides hiperbólicos de dos hojas (Fig. 2).

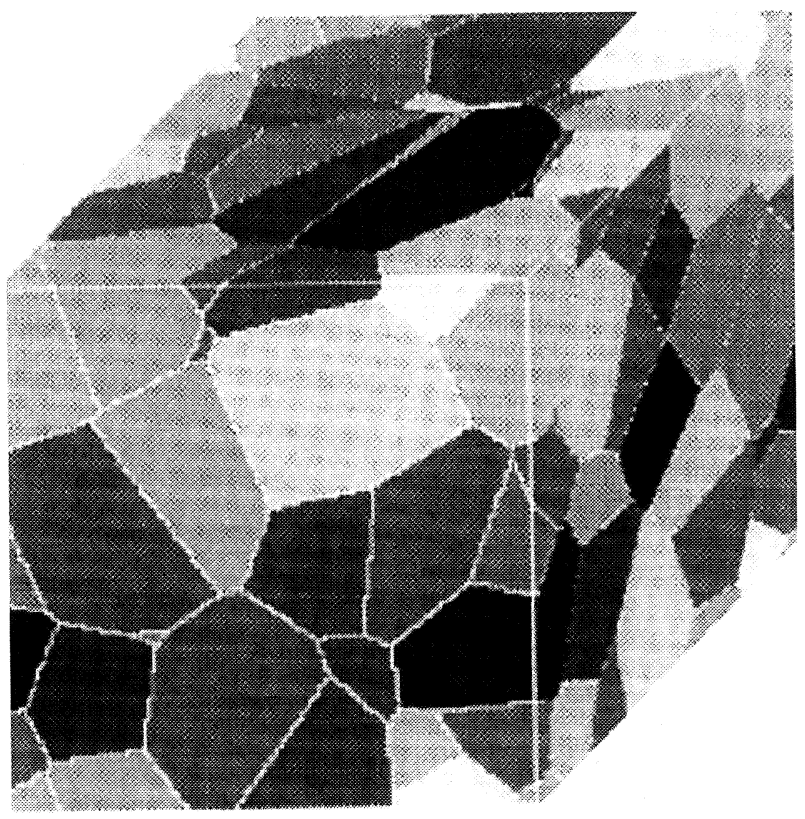

FIG. 1.- Un cubo descompuesto en $160 \times 160 \times$ 160 vóxeles. Teselación de Voronoi. La microestructura es periódica en las 3 direcciones perpendiculares. El cubo contiene 50 semillas dispuestas al azar. Los límites de grano son superficies planas.

FIG. 1.- A cube discretized in $160 \times 160 \times 160$ voxels. Voronoi tessellation from 50 nuclei (planar grain boundaries). Periodic boundary conditions.

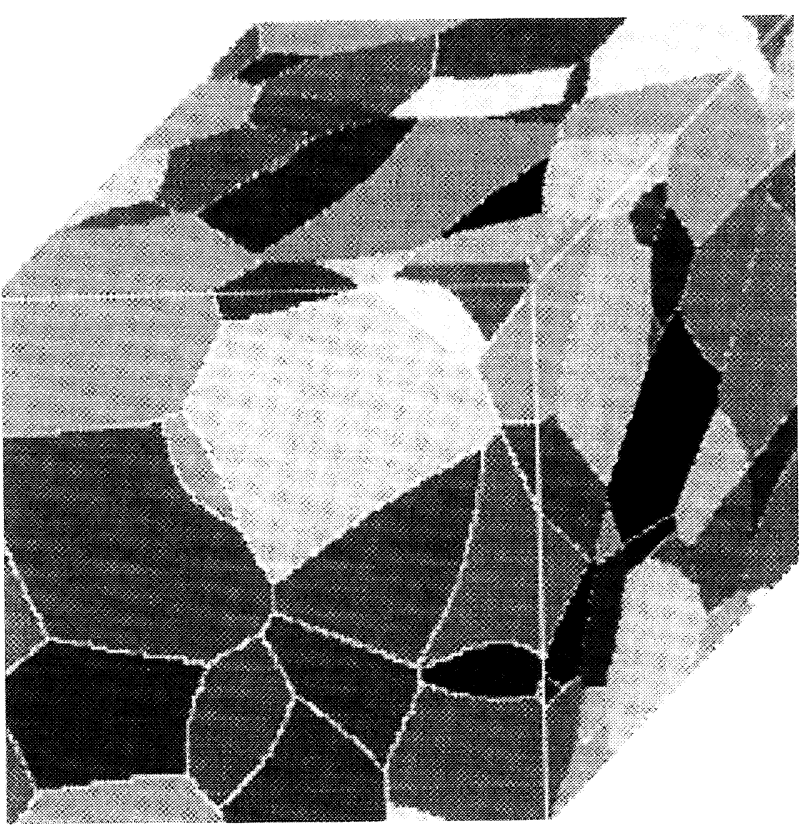

Fig. 2.- Un cubo descompuesto en $160 \times 160 \times$ 160 vóxeles tras realizar una teselación JMAK. En el interior del cubo se distribuyen al azar 50 semillas, en las mismas posiciones empleadas en la figura 1. La velocidad de nucleación de semillas es constante y, en el intervalo entre la nucleación de dos semillas consecutivas, los granos crecen una distancia igual a la mitad del lado de los vóxeles. Obsérvese que ahora los límites de grano son paraboloides hiperbólicos de dos hojas.

FIG. 2.- A cube discretized in $160 \times 160 \times 160$ voxels, JMAK tessellation from the same 50 nucleias in figure 1 (grain boundaries are now hyperbolic paraboloids). Constant nucleation equal to growth interval of half a voxel size.

Con este esquema resulta fácil imponer crecimientos preferenciales en determinadas direcciones de los granos. Por ejemplo, a cada semilla se le pueden asignar diferentes velocidades de crecimiento en 3 direcciones ortogonales. Las distancias se calculan ahora como el radio correspondiente en las coordenadas elípticas homofocales de la semilla en cuestión. El crecimiento de las distintas semillas puede especificarse con mucha flexibilidad: preferente en distintas direcciones al azar o compartiendo una dirección o plano preferente común, etc. Obviamente, también es posible realizar teselaciones incompletas imponiendo una distancia que limite el crecimiento de los distintos granos, o mediante otras hipótesis, creando, por ejemplo, estructuras multimodales.

\subsection{Teselaciones aleatorias}

Se selecciona un vóxel al azar, se averigua a qué granos pertenecen sus vóxeles vecinos y se asigna 
al grano con el que comparta un mayor número de vóxeles vecinos. Si el vóxel aún no tiene vecinos asignados a ningún grano, se selecciona otro vóxel al azar y se repite el proceso. La teselación resultante no es unívoca: es una teselación con resultados aleatorios que depende de la secuencia de selección de los vóxeles, la cual no es conocida de antemano. El resultado que se obtiene es semejante a una teselación de Voronoi, pero con unos límites de grano más irregulares (Fig. 3).

Un vóxel cúbico tiene 26 vóxeles vecinos de contacto: 6 vecinos de cara, 12 de lado y 8 de vértice. Estos vecinos pueden ponderarse, a la hora de establecer la preponderancia de vecindad, con igual peso o con pesos distintos. El programa habitualmente pondera con un peso de 1 a los vecinos de cara, de 0,5 a los vecinos de lado y de 0,25 a los de vértice. De este modo, el peso total de los vecinos es 14 , cifra que coincide con las caras de un tetrakaidecaedro de Kelvin (el poliedro que suele suponerse tesela el espacio con la mínima relación superficie/volumen)(6) .

Resulta aún posible asignar diferentes velocidades de crecimiento a las diferentes semillas. Para ello, una vez que se ha tomado la decisión de que este vóxel se asigna a determinado grano, se puede

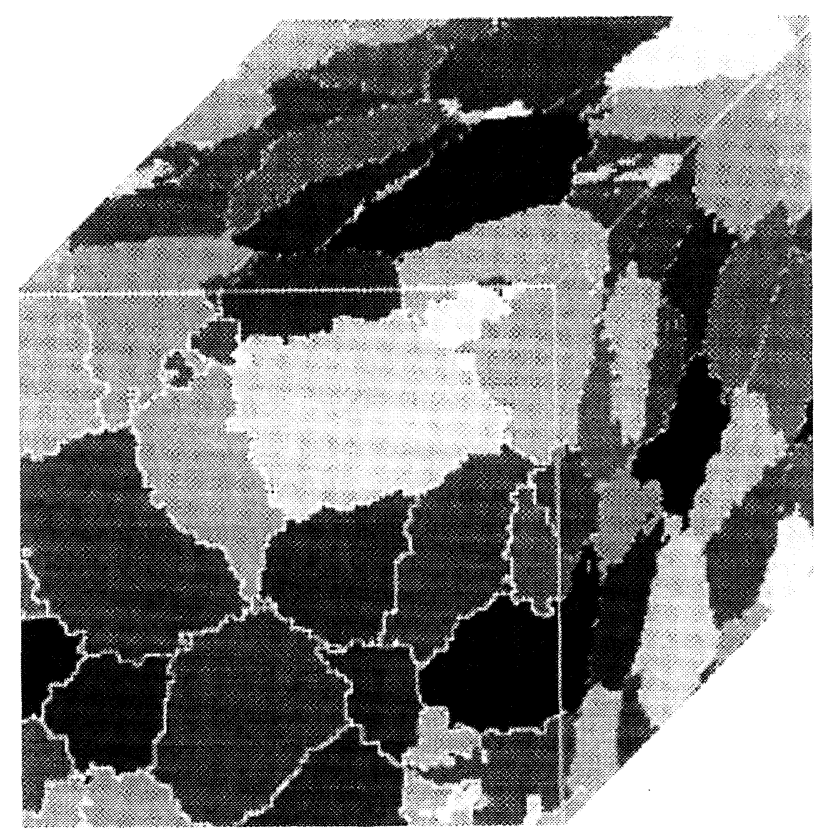

FIG. 3.- Un cubo descompuesto en $160 \times 160 \times$ 160 vóxeles tras realizar una teselación de Voronoi aleatoria. En el interior del cubo se han dispuesto al azar 50 semillas, en las mismas posiciones que las empleadas en la figura 1. Obsérvese que los límites de grano son ahora mucho más irregulares.

FIG. 3.- A cube discretized in $160 \times 160 \times 160$ voxels, random version of Voronoi tessellation from 50 nuclei. Note the roughness of grain boundaries. generar un nuevo número aleatorio y decidir si efectivamente se realiza o no la asignación.

Las semillas se pueden activar en el instante inicial de la simulación o se puede suponer una ley de nucleación dependiente del tiempo. Cuando un vóxel se haya intentado transformar un número prefijado de veces sin éxito, se define como nueva semilla; de esta segunda forma se obtienen teselaciones del tipo JMAK en 3D, pero con los límites de grano más irregulares que los de teselaciones JMAK deterministas.

\subsection{Condiciones de contorno}

Habitualmente, el programa genera microestructuras periódicas. Todas las caras del cubo continúan en su cara opuesta. En el caso de una teselación aleatoria, si el vóxel se sitúa cerca de la superficie del cubo, algunos vecinos se encontrarán en la cara opuesta del cubo. O, en el caso de las teselaciones matemáticas, las distancias se miden desde el vóxel a todas' las semillas y a las semejantes dispuestas en los otros 26 cubos, que se supone limitan el cubo que se está calculando.

Los tiempos de cálculo son relativamente largos, porque el número de distancias a calcular es muy elevado. Para cada vóxel (y normalmente hay $160 \times$ $160 \times 160=4.096 .000$ vóxeles), se calculan las distancias a las semillas de $27^{*}$ cubos con típicamente 50 semillas por cubo. En definitiva, se deben calcular 5.529.600.000 distancias (en el caso de una teselación determinística). El tiempo de cálculo es aún mayor en el caso de una teselación aleatoria, pues la asignación del vóxel a un determinado grano no está garantizada en cada intento, especialmente cuando la fracción en volumen asignada a granos es aún pequeña y la mayoría de los vóxeles carecen de granos vecinos.

\section{OTROS EJEMPLOS DE APLICACIÓN}

\subsection{Reconocimiento de fronteras de grano}

Tras completar las teselaciones, el programa reconoce los vóxeles situados en los límites de grano. Un vóxel es interior al grano si todos los vóxeles vecinos pertenecen al mismo grano que él. De lo contrario, pertenece a la frontera entre granos. La condición de pertenecer a la frontera puede relajarse perdonando alguna esquina o lado del vóxel. En definitiva, se puede fijar el peso total de

\footnotetext{
* La simetría permite reducir la búsqueda a un volumen equivalente a 8 cubos: el propio cubo, 6 medios cubos vecinos de cara, 12 cuartos de cubos vecinos de arista y 8 octavos de cubos vecinos de vértice.
} 
los vecinos que se desee para especificar cuándo el vóxel pertenece o no a la intercara entre los granos.

Una vez reconocidos los vóxeles pertenecientes a la intercara, el ordenador puede resembrar nuevas semillas sobre vóxeles asignados a intercaras y realizar una transformación de la estructura (de nuevo, de un modo determinista o aleatorio). Los nuevos granos podrán crecer (a uno o a ambos lados de la frontera) exclusivamente sobre los antiguos granos o a través de toda la microestructura. La figura 4 muestra el ejemplo de una recristalización nucleada sobre una sección de una previa teselación de Voronoi en tres dimensiones (la cara frontal de la figura 1). 1.500 nuevas semillas se han distribuido aleatoriamente sobre los vóxeles situados en límites de grano. La teselación posterior es de Voronoi.

\subsection{Transformaciones con morfología orientada}

La figura 5 representa un monocristal cúbico, donde 50 semillas se han distribuido al azar. Los granos crecen a partir de las semillas y su crecimiento es preferente sobre los planos $\{111\}$. Los crecimientos en las tres direcciones perpendiculares guardan la relación de 50/50/1; los dos primeros sobre los planos $\{111\}$ y el último en la dirección perpendicular. Cada semilla elige, al azar, uno de los 8 posibles planos $\{111\}$. Se trata, por ejemplo,

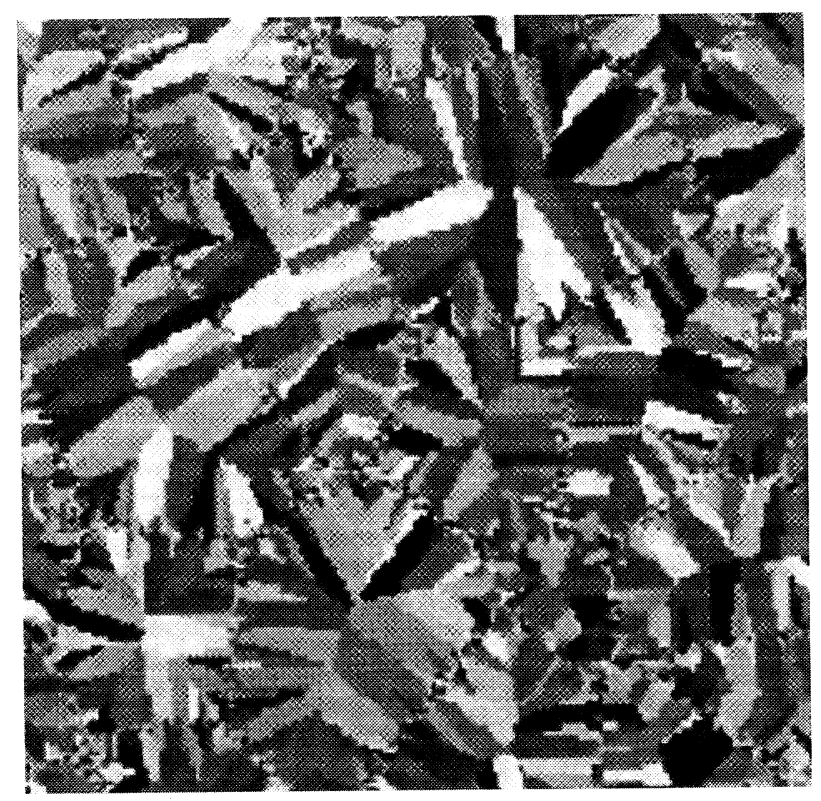

FIG. 4.- Sobre las juntas de grano de una sección de una teselación en 3D de Voronoi (la cara frontal de la figura 1) se han resembrado 1.500 nuevas semillas. La nueva teselación también se realiza de acuerdo con Voronoi.

FIG. 4.- Voronoi tessellation from 1,500 nuclei randomly located at the grain boundaries of the previous Voronoi structure of figure 1.
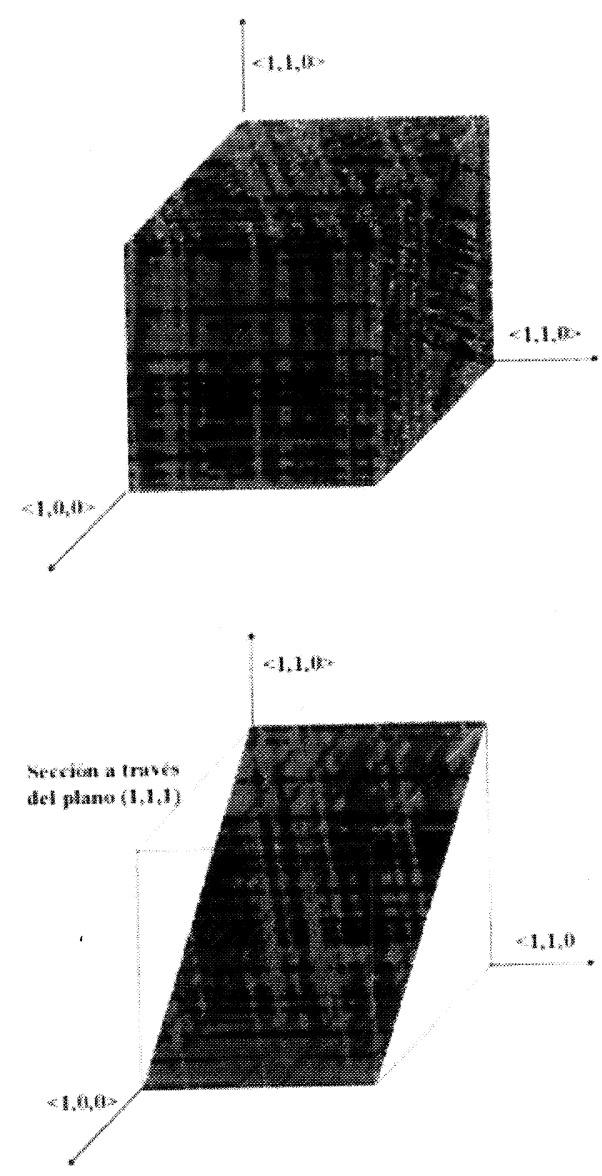

FIG. 5.- Sobre un monocristal cúbico se realiza una transformación a partir de 50 semillas distribuidas al azar. Los nuevos granos crecen preferentemente sobre los planos $\{111\}$. Las velocidades de crecimiento en las 3 direcciones perpendiculares guardan una proporción 50/50/1.

FIG. 5.- Transformation of a cube crystal with preferred growth on $\{111\}$ planes and from 50 nuclei randomly distributed in the volume. The ratio of growth rate is 50/50/1 on any direction on the preferred plane relative to the direction normal to it. The structure mimics the acicular ferrite structure or the original Widmanstätten structure of nickel-iron meteorites (octahedrite).

de una transformación ccc a cc, tal como la transformación (completa) de austenita en ferrita acicular, por nucleación intragranular, con plano de hábito $\{111\}_{\text {ccc }}$. La morfología obtenida reproduce fielmente la del constituyente "octaedrita" de muchos meteoritos metálicos (la estructura original de Widmanstätten).

\section{CONCLUSIONES}

Se ha desarrollado un programa de ordenador que genera microestructuras virtuales en 3D. 
Trabaja sobre elementos cúbicos de volumen (vóxeles) y eso simplifica los cálculos y los hace muy eficientes. El programa, incluso en su versión actual, es muy flexible y permite realizar cualquier teselación de tipo JMAK, incorporando diferentes velocidades de crecimiento o direcciones preferenciales del crecimiento.

Los ejemplos de estructuras simuladas ponen claramente de manifiesto que una estructura policristalina, incluso monofásica, no queda bien descrita especificando únicamente su tamaño de grano. Todas las microestructuras que se muestran en este artículo, con excepción de la figura 4, contienen el mismo número de granos (50) y topología aproximadamente similar (se han generado a partir de idénticos núcleos, incluso la figura 5). Como se observa, diferentes hipótesis de cálculo conducen a microestructuras muy diversas.

\section{Agradecimiento}

Este programa de ordenador ha sido desarrollado en el contexto de un proyecto de investigación europeo CECA (ECSC Project P3742), coordinado por el IRSID (Francia) y en colaboración con Hoogovens N. V. (Holanda), IEHK (Aquisgrán, Alemania) y CEIT (España).

\section{REFERENCIAS}

(1) Humphreys, F.J. y Hatherly, M. Recrystallization and Related Annealing Phenomena, Cap. 13, Pergamon Press, Oxford (R. U.), 1996.

(2) Underwood, E.E. Quantitative Stereology, AddisonWesley Publ. Comp., Reading (Mass. EE, UU.), 1970.

(3) Marthinsen, K. y Ryum, N. Transformation kinetics and microstructure for grain boundary nucleated recrystallization in two dimensions", Acta Mater., 45, (3), 1997: 1.127-1.136.

(4) Ripley, B.D. Stochastic Simulation, John Wiley \& Sons, Londres (R. U.), 1986.

(5) Stoyan, D., Kendall, W.S. y Mecke, J. Stochastic Geometry and its Applications, $2^{\mathrm{a}}$ edc., John Wiley \& Sons, Chichester (R. U.), 1985.

(6) Weaire, D. The Kelvin Problem: Foam Structures of Minimal Surface Area, Taylor \& Francis Publ. Comp. Inc., Londres (R. U.), 1998. 